\title{
Structural Modification of the Macrolide Brefeldin A to Analogues with Enhanced Cytotoxicity against KB Cells
}

\author{
Maneekarn Namsa-Aid ${ }^{1, *}$, Suthep Wiyakrutta ${ }^{2}$, Surasak Prachya ${ }^{3}$, \\ Anucha Namsa-Aid ${ }^{3}$ and Apichart Suksamrarn ${ }^{4}$ \\ ${ }^{I}$ Department of Chemistry and Center of Excellence for Innovation in Chemistry, Faculty of Science, \\ Srinakharinwirot University, Bangkok 10110, Thailand \\ ${ }^{2}$ Department of Microbiology, Faculty of Science, Mahidol University, Bangkok 10400, Thailand \\ ${ }^{3}$ Laboratory of Natural Products, Chulabhorn Research Institute, Bangkok 10210, Thailand \\ ${ }^{4}$ Department of Chemistry, Faculty of Science, Ramkhamhaeng University, Bangkok 10240, Thailand
}

('Corresponding author's e-mail: maneekarn@g.swu.ac.th)

Received: 30 June 2020, Revised: 16 May 2021, Accepted: 30 May 2021

\begin{abstract}
The macrolide brefeldin A (BFA, 1) exhibited high cytotoxicity against KB cells. However, it was also toxic against non-cancerous cells. In order to lower toxicity against normal cells while maintaining the cytotoxic potency to the cancer cells, structural modification of this compound was undertaken. Starting from compound 1, the analogues 2-13 were synthesized and evaluated for cytotoxicity against $\mathrm{KB}$ cells. The analogue 2 exhibited the most potent cytotoxicity against $\mathrm{KB}$ cells, with an $\mathrm{IC}_{50}$ value of $0.034 \mathrm{nM}, 67$-fold more active than its parent compound 1. It was 41764 and 8235 fold more active than the standard drugs ellipticine and doxorubicin, respectively. The higher cytotoxicity against KB cells and lower toxicity against Vero cells of analogue $\mathbf{2}$ than those of the parent compound $\mathbf{1}$ contributed to its exceptionally high selectivity index of 9117 . The results suggested that this analogue might be utilized to develop a new candidate for an anticancer drug.
\end{abstract}

Keywords: Brefeldin A, Synthetic analogues, Cytotoxicity, Bioactivity

\section{Introduction}

Brefeldin A (BFA, 1) is a macrolide antibiotic that was first isolated from Penicillium decumbens under the name decumbin [1] and later from various fungi such as P. brefeldianum [2,3], P. cyaneum [4], Ascochyta imperfect [5], and other sources [6-10]. Initially, it was recognized that BFA has a wide range of antibiotic activities [2,11] and has been studied as a potential anticancer [12-17] and antiviral prodrug [13]. BFA was characterized as an inhibitor that uncompetitively targets protein/protein interface and stabilizes the complex in an abortive conformation causing blockage of membrane traffic at the Golgi apparatus $[18,19]$.

Oral cancer is a major worldwide public health issue and may affect any oral cavity region, including the lips, tongue, mouth and throat. Globally, an estimated 377,713 new cancer cases and 177,757 cancer deaths occurred in 2020 [20]. In Thailand, oral cancer reported at $2.5 \%$ for new cases and $2.0 \%$ for oral cancer deaths, and was ranked the $10^{\text {th }}$ frequent cancer [21]. The high level of mortality was from late diagnosis, in which the patients went through the advanced or metastatic stage. Although standard therapies such as radiotherapy and chemotherapy are suitable for oral cancer treatment, there are still several issues that need to be considered such as side effects from the treatment, drug resistance, and the high expense of treatment [22]. Therefore, the development of chemotherapeutic agents may reduce side effects and improving treatment for oral cancer.

Previous investigations on the structure-activity relationship of BFA indicated that the ability to induce DNA fragmentation and apoptosis in human colonic carcinoma cell line HTC116 required the conformational rigidity of the molecule, the $\alpha, \beta$-unsaturated lactone moiety, the carbon-carbon double bonds, and the configuration of the 4-hydroxyl group [23,24]. Oxidation of the C7 hydroxyl group of BFA resulted in an increase in antifungal activity against Candida albicans [25], while oxidation and methylation of the C4 and C7 hydroxyl groups reduced the cytotoxic activity [23,24]. Microbial glycosylation and acetylation of BFA markedly lowered its cytotoxicity and antifungal activity [26]. 
Sulfide derivatives obtained via the Michael addition of various thiols to the $\alpha, \beta$-unsaturated lactone of BFA were shown to have enhanced aqueous solubility relative to BFA itself. The sulfides could be considered as BFA prodrugs since, in the biological system, they were metabolically oxidized to sulfoxide, and underwent subsequent syn elimination to regenerate the BFA $[15,16]$. In 2006, ester derivatives of BFA were synthesized and their antiproliferative activity was determined. Monoderivatization was reported to retain the biological activity, whereas the diester analogues were the least active [27]. The presence of the methyl glutarates was more cytotoxic than their acid counterparts. Nitric oxide-donating mono- or diester derivatives of brefeldin A were synthesized and evaluated for antiproliferative activity. Some of them displayed potent cytotoxicity against human prostate cancer PC-3 cells, human colon carcinoma HT-29 cells and human liver cancer HepG-2 cells [28]. Acylated BFA derivatives were synthesized under mild conditions and showed potent antitumor activity against TE-1 cell. The 7-O-benzoate BFA, 4,7-O-dibenzoate BFA, and 7-O-biotin carboxylate BFA were described as the most potent cytotoxic activity [29]. Nonpolar substituent at C15 of BFA exhibited significant cytostatic effects in human cancer cells [30].

However, these analogues were less cytotoxic than BFA. Our preliminary work discovered that the presence of an ester function with aliphatic side chain resulted in increased cytotoxicity against certain cancer cell lines. Thus, it was interesting to investigate whether such an ester moiety would increase cytotoxicity with respect to the BFA. The present work describes the cytotoxicity of BFA and its analogues. The chemical modifications described in this paper were focused on the preparations of mono and diester derivatives of BFA with various alkyl and aromatic side chains, and synthesis of analogues with modified functional group in the molecule of BFA. This is the first report of the cytotoxicity against $\mathrm{KB}$ cells of these analogues.

\section{Materials and methods}

${ }^{1} \mathrm{H}$ - and ${ }^{13} \mathrm{C}-\mathrm{NMR}$ spectra were recorded on a Bruker AVANCE 300 FT-NMR spectrometer, operating at $300 \mathrm{MHz}\left({ }^{1} \mathrm{H}\right)$ and $75 \mathrm{MHz}\left({ }^{13} \mathrm{C}\right)$. ESITOF-MS was obtained from a Micromass LCT mass spectrometer. Column chromatography and TLC were carried out using Merck silica gel 60 ( $>230$ mesh) and precoated silica gel 60 F254 plates, respectively. Brefeldin A (BFA, 1) was isolated from the endophytic fungus KLAR 5 belonging to order Hypocreales [6]. The reactions were monitored by TLC and by spraying with anisaldehyde- $\mathrm{H}_{2} \mathrm{SO}_{4}$ reagent followed by heating.

\section{Synthesis of 7-O-acetylBFA (2), 4-O-acetylBFA (3), and 4,7-di- $O$-acetylBFA (7)}

A mixture of BFA $(1,10 \mathrm{mg})$ and acetic acid was refluxed for $4 \mathrm{~h}$. After the completion of the esterification, water was added, and the solution mixture was extracted 3 times with ethyl acetate. The combined organic layer was washed with water and dried over anhydrous $\mathrm{Na}_{2} \mathrm{SO}_{4}$. The solvent was evaporated and the residue was chromatographed on a silica column by elution with $\mathrm{CH}_{2} \mathrm{Cl}_{2} / \mathrm{MeOH}$ (99:1) to afford 7-O-acetylBFA (2, $4.6 \mathrm{mg}, 40 \%)$, 4- $O$-acetylBFA (3, $2.3 \mathrm{mg}, 20 \%)$ and 4,7-di- $O$ acetylBFA (7, $3.4 \mathrm{mg}, 26 \%)$.

7-O-AcetylBFA (2). Yield $40 \%,{ }^{1} \mathrm{H}-\mathrm{NMR}$ spectrum $\left(300 \mathrm{MHz}, \mathrm{CDCl}_{3}, \delta, \mathrm{ppm}, J / \mathrm{Hz}\right) ; 0.97(1 \mathrm{H}, \mathrm{m}$, H-13a), 1.28 (3H, d, $J=6.3, \mathrm{H}-16), 1.52$ - 1.65 (3H, m, H-13b, H-14), 1.74 - 1.94 (5H, m, H-5, H-6, H8a, H-12a), 2.05 (3H, s, H-2'), 2.22 (1H, m, H-8b), 2.31 (1H, m, H-12b), $2.40(1 \mathrm{H}, \mathrm{m}, \mathrm{H}-9), 4.15$ (1H, m, $\mathrm{H}-4), 4.89(1 \mathrm{H}, \mathrm{m}, \mathrm{H}-15), 5.16(1 \mathrm{H}, \mathrm{m}, \mathrm{H}-7), 5.24(1 \mathrm{H}, \mathrm{dd}, J=9.3,15.1, \mathrm{H}-10), 5.74(1 \mathrm{H}, \mathrm{ddd}, J=4.8$, 10.2, 15.1, H-11), 5.94 (1H, dd, $J=1.8,15.7, \mathrm{H}-2), 7.36(1 \mathrm{H}, \mathrm{dd}, J=3.3,15.7, \mathrm{H}-3) .{ }^{13} \mathrm{C}-\mathrm{NMR}(75 \mathrm{MHz}$, $\left.\mathrm{CDCl}_{3}, \delta, \mathrm{ppm}\right) 20.8$ (q, C-16), 21.3 (q, C-2'), 26.6 (t, C-13), 31.8 (t, C-12), 34.1 (t, C-14), 38.5 (t, C-6), 40.1 (t, C-8), 44.0 (d, C-9), 52.1 (d, C-5), 71.7 (d, C-15), 75.1 (d, C-7), 75.9 (d, C-4), 117.8 (d, C-2), 131.0 (d, C-11), 135.9 (d, C-10), 151.2 (d, C-3), 166.1 (s, C-1), 170.1 (s, C-1'). Mass spectrum (HRESITOF-MS) $m / z: 345.1667$ (Calcd for $\mathrm{C}_{18} \mathrm{H}_{26} \mathrm{O}_{5} \mathrm{Na}: 345.1672$ ).

4-O-AcetylBFA (3) Yield $20 \%,{ }^{1} \mathrm{H}-\mathrm{NMR}\left(300 \mathrm{MHz}, \mathrm{CDCl}_{3}, \delta\right.$, ppm, J/Hz) $0.95(1 \mathrm{H}, \mathrm{m}, \mathrm{H}-13 \mathrm{a})$, 1.26 (3H, d, $J=6.3, \mathrm{H}-16), 1.48-2.05$ (9H, m, H-5, H-6, H-8a, H-12, H-13b, H-14), 2.14 (3H, s, H-2'), 2.24 (1H, m, H-8b), 2.44 (1H, m, H-9), 4.34 (1H, m, H-7), 4.88 (1H, m, H-15), 5.30 (2H, m, H-4, H-10), $5.72(1 \mathrm{H}, \mathrm{m}, \mathrm{H}-11), 5.73(1 \mathrm{H}, \mathrm{dd}, J=1.8,15.7, \mathrm{H}-2), 7.26(1 \mathrm{H}, \mathrm{dd}, J=3.3,15.7, \mathrm{H}-3) .{ }^{13} \mathrm{C}-\mathrm{NMR}(75$ $\mathrm{MHz}, \mathrm{CDCl}_{3}, \delta$, ppm) 20.7 (q, C-2'), 20.8 (q, C-16), 26.6 (t, C-13), 31.8 (t, C-12), 34.1 (t, C-14), 41.0 (t, C6), 43.3 (t, C-8), 44.3 (d, C-9), 49.5 (d, C-5), 71.8 (d, C-7), 72.4 (d, C-15), 76.4 (d, C-4), 118.3 (d, C-2), 130.7 (d, C-11), 136.3 (d, C-10), 147.2 (d, C-3), 165.5 (s, C-1), 170.0 (s, C-1'). Mass spectrum (HRESITOF-MS) $m / z: 345.1674$ (Calcd for $\mathrm{C}_{18} \mathrm{H}_{26} \mathrm{O}_{5} \mathrm{Na}: 345.1672$ ).

4,7-di-O-AcetylBFA (7) Yield $26 \%,{ }^{1} \mathrm{H}-\mathrm{NMR}\left(300 \mathrm{MHz}, \mathrm{CDCl}_{3}, \delta, \mathrm{ppm}, J / \mathrm{Hz}\right) 0.96(1 \mathrm{H}, \mathrm{m}, \mathrm{H}-$ 13a), 1.27 (3H, d, J=6.3, H-16), 1.53 - 1.91 (9H, m, H-5, H-6, H-8a, H-12, H-13b, H-14), 2.05 (3H, s, 
H-2"), 2.33 (1H, m, H-8b), 2.47 (1H, m, H-9), 2.55 (3H, s, H-2'), 4.88 (1H, m, H-15), 5.14 (1H, m, H-7), $5.25(2 \mathrm{H}$, overlapping $\mathrm{m}$ and $\mathrm{dd}, \mathrm{H}-4, \mathrm{H}-10), 5.72(1 \mathrm{H}, \mathrm{dd}, J=1.8,15.7, \mathrm{H}-11), 5.74(1 \mathrm{H}, \mathrm{m}, \mathrm{H}-2), 7.25$ $(1 \mathrm{H}, \mathrm{dd}, J=3.4,15.3, \mathrm{H}-3) .{ }^{13} \mathrm{C}-\mathrm{NMR}\left(75 \mathrm{MHz}, \mathrm{CDCl}_{3}, \delta, \mathrm{ppm}\right) 20.7$ (q, C-2"), 20.8 (q, C-2'), 21.3 (q, C16), 26.5 (t, C-13), 31.8 (t, C-12), 34.1 (t, C-14), 38.3 (t, C-6), 40.1 (t, C-8), 44.1 (d, C-9), 49.7 (d, C-5), 71.8 (d, C-15), 75.2 (d, C-7), 76.3 (d, C-4), 118.4 (d, C-2), 131.2 (d, C-11), 135.7 (d, C-10), 147.1 (d, C3), 165.6 (s, C-1), 169.8 (s, C-1'), 170.6 (s, C-1"). Mass spectrum (HR-ESITOF-MS) m/z: 387.1776 (Calcd for $\mathrm{C}_{20} \mathrm{H}_{28} \mathrm{O}_{6} \mathrm{Na}: 387.1778$ ).

\section{General procedure for the synthesis of 4- $O$-alkyl and 4,7-di- $O$-alkyl esters of BFA}

A stirred solution of BFA $(1,10 \mathrm{mg})$ in pyridine $(0.5 \mathrm{~mL})$ was added to acid anhydride, and the reaction mixture was stirred overnight. After the completion of the esterification, water was added, and the solution mixture was extracted 3 times with ethyl acetate. The combined organic layer was washed with water and dried over anhydrous $\mathrm{Na}_{2} \mathrm{SO}_{4}$. The solvent was then evaporated and the residue was chromatographed on a silica column by elution with $\mathrm{CH}_{2} \mathrm{Cl}_{2} / \mathrm{MeOH}(99: 1)$ to afford 4-O-alkyl and 4,7-di$O$-alkyl esters of BFA.

4-O-PropanoylBFA (4) ${ }^{1} \mathrm{H}-\mathrm{NMR}\left(300 \mathrm{MHz}, \mathrm{CDCl}_{3}, \delta\right.$, ppm, J/Hz) $0.96(1 \mathrm{H}, \mathrm{m}, \mathrm{H}-13 \mathrm{a}), 1.18(3 \mathrm{H}$, $\mathrm{t}, J=7.5, \mathrm{H}-3$ ') 1.26 (3H, d, $J=6.3, \mathrm{H}-16), 1.51-2.03$ (8H, m, H-6, H-8a, H-12, H-13b, H-14), 2.20 (2H, m, H-5, H-8b), 2.42 (2H, q, J=7.5, H-2'), 2.43 (1H, m, H-9), 4.33 (1H, m, H-7), 4.87 (1H, m, H-15), $5.31(2 \mathrm{H}, \mathrm{m}, \mathrm{H}-4, \mathrm{H}-10), 5.71(1 \mathrm{H}, \mathrm{dd}, J=1.8,15.8, \mathrm{H}-11), 5.72(1 \mathrm{H}, \mathrm{m}, \mathrm{H}-2), 7.27(1 \mathrm{H}, \mathrm{dd}, J=3.3$, 15.8, H-3). ${ }^{13} \mathrm{C}-\mathrm{NMR}\left(75 \mathrm{MHz}, \mathrm{CDCl}_{3}, \delta\right.$, ppm) 9.2 (q, C-3'), 20.8 (q, C-16), 26.6 (t, C-13), 27.6 (t, C-2'), 31.8 (t, C-12), 34.1 (t, C-14), 40.9 (t, C-6), 43.3 (t, C-8), 44.4 (d, C-9), 49.5 (d, C-5), 71.8 (d, C-7), 72.4 (d, C-15), 76.1 (d, C-4), 118.2 (d, C-2), 130.7 (d, C-11), 136.3 (d, C-10), 147.4 (d, C-3), 165.8 (s, C-1), 173.4 (s, C-1'). Mass spectrum (HR-ESITOF-MS) $m / z$ : 359.1829 (Calcd for $\mathrm{C}_{19} \mathrm{H}_{28} \mathrm{O}_{5} \mathrm{Na}$ : 359.1829).

4-O-ButanoylBFA (5) ${ }^{1} \mathrm{H}-\mathrm{NMR}\left(300 \mathrm{MHz}, \mathrm{CDCl}_{3}, \delta\right.$, ppm, $\left.J / \mathrm{Hz}\right) 0.99(3 \mathrm{H}, \mathrm{t}, J=7.4, \mathrm{H}-4$ '), 1.26 $(3 \mathrm{H}, \mathrm{d}, J=6.3, \mathrm{H}-16), 1.71$ (2H, m, H-3'), $1.78-2.04$ (9H, m, H-5, H-6, H-12, H-13, H-14), 2.22 (2H, m, H-8), 2.37 (2H, t, $\left.J=7.5, \mathrm{H}-2^{\prime}\right), 2.41(1 \mathrm{H}, \mathrm{m}, \mathrm{H}-9), 4.34$ (1H, m, H-7), 4.87 (1H, m, H-15), 5.31 (2H, m, $\mathrm{H}-4, \mathrm{H}-10), 5.71(1 \mathrm{H}, \mathrm{dd}, J=1.8,15.6, \mathrm{H}-2), 5.73(1 \mathrm{H}, \mathrm{m}, \mathrm{H}-11), 7.28(1 \mathrm{H}, \mathrm{dd}, J=3.4,15.6, \mathrm{H}-3) .{ }^{13} \mathrm{C}-$ NMR (75 MHz, $\left.\mathrm{CDCl}_{3}, \delta, \mathrm{ppm}\right) 13.7$ (q, C-4'), 18.5 (t, C-3'), 20.8 (q, C-16), 26.6 (t, C-13), 31.8 (t, C-12), 34.1 (t, C-14), 36.2 (t, C-2'), 41.0 (t, C-6), 43.3 (t, C-8), 44.3 (d, C-9), 49.6 (d, C-5), 71.8 (d, C-7), 72.4 (d, C-15), 76.0 (d, C-4), 118.2 (d, C-2), 130.7 (d, C-11), 136.4 (d, C-10), 147.5 (d, C-3), 165.9 (s, C-1), 172.5 (s, C-1'). Mass spectrum (HR-ESITOF-MS) $m / z$ : 373.1981 (Calcd for $\mathrm{C}_{20} \mathrm{H}_{30} \mathrm{O}_{5} \mathrm{Na}: 373.1985$ ).

4-O-BenzoylBFA (6) ${ }^{1} \mathrm{H}-\mathrm{NMR}\left(300 \mathrm{MHz}, \mathrm{CDCl}_{3}, \delta\right.$, ppm, $\left.\mathrm{J} / \mathrm{Hz}\right) 0.99(1 \mathrm{H}, \mathrm{m}, \mathrm{H}-13 \mathrm{a}), 1.27(3 \mathrm{H}, \mathrm{d}$, $J=6.3, \mathrm{H}-16), 1.58$ (3H, m, H-8a, H-14), 1.76 (2H, m, H-6a, H-13b), 1.89 (1H, m, H-12a), 2.02 (2H, m, H-6b, H-12b), 2.29 (1H, m, H-8b), 2.38 (1H, m, H-5), 2.53 (1H, m, H-9), 4.37 (1H, m, H-7), 4.88 (1H, m, $\mathrm{H}-15), 5.37$ (1H, dd, $J=9.6,15.1, \mathrm{H}-10), 5.78(1 \mathrm{H}, \mathrm{m}, \mathrm{H}-11), 5.54$ (1H, m, H-4), 5.80 (1H, dd, J = 1.8, 15.6, H-2), 7.38 (2H, dd, $J=3.3,15.6, \mathrm{H}-3), 7.49$ (2H, t, J = 7.5, H-4', H-4"), 7.62 (1H, br. t, J= 7.2, H$\left.5^{\prime}\right), 8.10\left(2 \mathrm{H}, \mathrm{dd}, \mathrm{J}=0.9,7.2, \mathrm{H}-3\right.$ ', H-3"), ${ }^{13} \mathrm{C}-\mathrm{NMR}(75 \mathrm{MHz}, \mathrm{CDCl} 3, \delta$, ppm) 20.8 (q, C-16), 26.6 (t, C13), 31.8 (t, C-12), 34.1 (t, C-14), 41.2 (t, C-6), 43.3 (t, C-8), 44.4 (d, C-9), 49.8 (d, C-5), 71.8 (d, C-7), 72.5 (d, C-15), 76.9 (d, C-4), 118.4 (d, C-2), 128.5 (2C, d, C-3', C-3"), 129.7 (2C, d, C-4', C-4"), 130.2 (s, C-2'), 130.7 (d, C-11), 133.3 (d, C-5'), 136.4 (d, C-10), 147.2 (d, C-3), 165.5 (s, C-1), 165.9 (s, C-1'). Mass spectrum (HR-ESITOF-MS) $m / z$ : 407.1828 (Calcd for $\mathrm{C}_{23} \mathrm{H}_{28} \mathrm{O}_{5} \mathrm{Na}$ : 407.1829).

4,7-di-O-PropanoylBFA (8) ${ }^{1} \mathrm{H}-\mathrm{NMR}\left(300 \mathrm{MHz}, \mathrm{CDCl}_{3}, \delta\right.$, ppm, $\left.J / \mathrm{Hz}\right) 0.96(1 \mathrm{H}, \mathrm{m}, \mathrm{H}-13 \mathrm{a}), 1.15$ $(3 \mathrm{H}, \mathrm{t}, J=7.5, \mathrm{H}-3 "), 1.18$ (3H, t, $J=7.5, \mathrm{H}-3$ '), 1.26 (3H, d, $J=6.3, \mathrm{H}-16), 1.48-1.90$ (6H, m, H-6, H8a, H-13b, H-14), $1.99-2.16$ (3H, m, H-5, H-12), 2.31 (1H, m, H-8b), 2.32 (2H, q, J= 7.5, H-2"), 2.41 (2H, q, J=7.5, H-2'), 2.47 (1H, m, H-9), 4.89 (1H, m, H-7), 5.14 (1H, m, H-15), 5.25 (2H, m, H-4, H-10), $5.70(1 \mathrm{H}, \mathrm{dd}, J=1.8,15.6, \mathrm{H}-2), 5.74(1 \mathrm{H}, \mathrm{m}, \mathrm{H}-11), 7.27(1 \mathrm{H}, \mathrm{dd}, J=3.3,15.6, \mathrm{H}-3) .{ }^{13} \mathrm{C}-\mathrm{NMR}(75$ $\mathrm{MHz}, \mathrm{CDCl} 3, \delta$, ppm) 9.1 (2C, q, 3", 3'), 20.7 (q, C-16), 26.5 (t, C-13), 27.5 (t, C-2"), 27.8 (t, C-2'), 31.8 (t, C-12), 34.1 (t, C-14), 38.3 (t, C-6), 40.1 (t, C-8), 44.1 (d, C-9), 49.8 (d, C-5), 71.8 (d, C-15), 75.0 (d, C-7), 76.1 (d, C-4), 118.3 (d, C-2), 131.1 (d, C-11), 135.8 (d, C-10), 147.3 (d, C-3), 165.7 (s, C-1), 173.3 (s, C-1"), 174.0 (s, C-1'). Mass spectrum (HR-ESITOF-MS) m/z: 415.2101 (Calcd for $\mathrm{C}_{22} \mathrm{H}_{32} \mathrm{O}_{6} \mathrm{Na}$ : 415.2091).

4,7-di-O-ButanoylBFA (9) ${ }^{1} \mathrm{H}-\mathrm{NMR}\left(300 \mathrm{MHz}, \mathrm{CDCl}_{3}, \delta\right.$, ppm, $\left.J / \mathrm{Hz}\right) 0.96(3 \mathrm{H}, \mathrm{t}, J=7.4, \mathrm{H}-4$ '), 0.98 (3H, t, $J=7.4, \mathrm{H}-4 "), 1.26$ (3H, d, $J=6.3, \mathrm{H}-16), 1.54-1.91$ (12H, m, H-6a, H-8, H-12a, H-13, H14, H-3', H-3"), 2.08 (3H, m, H-5, H-6b, H-12b), 2.28 (2H, t, J= 7.5, H-2'), 2.36 (2H, t, J= 7.5, H-2"), 2.46 (1H, m, H-9), 4.88 (1H, m, H-7), 5.15 (1H, m, H-15), 5.24 (1H, m, H-10), 5.30 (1H, m, H-4), 5.71 $(1 \mathrm{H}, \mathrm{dd}, J=1.9,15.6, \mathrm{H}-2), 5.74(1 \mathrm{H}, \mathrm{m}, \mathrm{H}-11), 7.27(1 \mathrm{H}, \mathrm{dd}, J=3.4,15.6, \mathrm{H}-3) .{ }^{13} \mathrm{C}-\mathrm{NMR}(75 \mathrm{MHz}$, $\left.\mathrm{CDCl}_{3}, \delta, \mathrm{ppm}\right) 13.6$ (q, C-4"), 13.7 (q, C-4'), 18.5 (2C, t, C-3', C-3"), 20.8 (q, C-16), 26.6 (t, C-13), 31.8 
(t, C-12), 34.1 (t, C-14), 36.1 (t, C-2"), 36.5 (t, C-2'), 38.4 (t, C-6), 40.1 (t, C-8), 44.1 (d, C-9), 49.9 (d, C5), 71.8 (d, C-7), 75.0 (d, C-15), 76.0 (d, C-4), 118.3 (d, C-2), 131.1 (d, C-11), 135.8 (d, C-10), 147.4 (d, C-3), 166.0 (s, C-1), 169.0 (s, C-1"), 172.5 (s, C-1'). Mass spectrum (HR-ESITOF-MS) m/z: 443.2414 (Calcd for $\mathrm{C}_{24} \mathrm{H}_{36} \mathrm{O}_{6} \mathrm{Na}$ : 443.2404).

4,7-di-O-BenzoylBFA (10) ${ }^{1} \mathrm{H}-\mathrm{NMR}\left(300 \mathrm{MHz}, \mathrm{CDCl}_{3}, \delta\right.$, ppm, J/Hz) $0.98(1 \mathrm{H}, \mathrm{m}, \mathrm{H}-13 \mathrm{a}), 1.28$ (3H, d, J=6.3, H-16), 1.58 (1H, m, H-14a), 1.73 - 1.96 (5H, m, H-6a, H-8a, H-12a, H-13b, H-14b), 2.06 (1H, m, H-12b), 2.27 - 2.55 (3H, m, H-5, H-6b, H-8b), 2.64 (1H, m, H-9), 4.90 (1H, m, H-15), 5.36 (1H, $\mathrm{dd}, J=9.6,5.0, \mathrm{H}-10), 5.44(1 \mathrm{H}, \mathrm{m}, \mathrm{H}-7), 5.58(1 \mathrm{H}, \mathrm{m}, \mathrm{H}-4), 5.82(1 \mathrm{H}, \mathrm{m}, \mathrm{H}-2), 5.84(1 \mathrm{H}, \mathrm{dd}, J=1.8$, 15.6, H-11), 7.41 (1H, dd, $J=3.3,15.6, \mathrm{H}-3), 7.48$ (4H, br. t, $J=7.8$, H-4', H-6', H-4", H-6"), 7.60 (2H, m, H-5', H-5"), 8.04 (2H, dd, $J=1.5,7.7, \mathrm{H}-3 ", \mathrm{H}-7 "), 8.09$ (2H, dd, $\left.J=1.5,7.7, \mathrm{H}-3{ }^{\prime}, \mathrm{H}-7{ }^{\prime}\right) .{ }^{13} \mathrm{C}-\mathrm{NMR}$ $\left(75 \mathrm{MHz}, \mathrm{CDCl}_{3}, \delta\right.$, ppm) 20.8 (q, C-16), 26.6 (t, C-13), 31.8 (t, C-12), 34.1 (t, C-14), 38.6 (t, C-6), 40.2 (t, C-8), 44.3 (d, C-9), 50.3 (d, C-5), 71.9 (d, C-15), 76.0 (d, C-7), 76.8 (d, C-4), 118.6 (d, C-2), 128.4 (2C, d, C-4", C-6"), 128.5 (2C, d, C-4', C-6'), 129.5 (2C, d, C-3", C-7"), 129.7 (2C, d, C-3', C-7'), 130.2 (2C, s, C-2', C-2"), 131.3 (d, C-11), 133.0 (d, C-5"), 133.4 (d, C-5'), 135.8 (d, C-10), 147.1 (d, C-3), 165.4 (s, C-1), 165.6 (s, C-1"), 166.0 (s, C-1'). Mass spectrum (HR-ESITOF-MS) m/z: 511.2081 (Calcd for $\left.\mathrm{C}_{30} \mathrm{H}_{32} \mathrm{O}_{6} \mathrm{Na}: 511.2091\right)$.

\section{Synthesis of 7-oxo-BFA (11) and 5,6-dehydro-2,3-dihydro-4,7-dioxo-BFA (12)}

A stirred solution of BFA (1, $10 \mathrm{mg})$ in dichloromethane $(1 \mathrm{~mL})$ was added to pyridinium chlorochromate. The reaction mixture was stirred for $4 \mathrm{~h}$ and was worked up in the usual fashion. The solvent was then evaporated and the residue chromatographed as described previously to afford 7-oxoBFA (11, $1.8 \mathrm{mg}, 18 \%)$ and 5,6-dehydro-2,3-dihydro-4,7-dioxo-BFA (12, $4.1 \mathrm{mg}, 42 \%)$.

7-Oxo-BFA (11) Yield $18 \%$. ${ }^{1} \mathrm{H}-\mathrm{NMR}\left(300 \mathrm{MHz}, \mathrm{CDCl}_{3}, \delta\right.$, ppm, $\left.J / \mathrm{Hz}\right) 1.06(1 \mathrm{H}, \mathrm{m}, \mathrm{H}-13 \mathrm{a}), 1.29$ (3H, d, J=6.3, H-16), 1.71 - 1.94 (5H, m, H-6a, H-12a, H-13b, H-14), 2.04 - 2.27 (3H, m, H-5, H-8a, H12b), 2.55 (1H, m, H-8b), 2.69 (1H, m, H-9), 2.85 (1H, m, H-6b), 4.26 (1H, br. d, J=8.4, H-4), 4.93 (1H, m, H-15), $5.23(1 \mathrm{H}, \mathrm{dd}, J=9.3,15.2, \mathrm{H}-10), 5.84(1 \mathrm{H}, \mathrm{ddd}, J=5.4,9.4,15.2, \mathrm{H}-11), 5.98(1 \mathrm{H}, \mathrm{dd}, J=$ $1.8,15.7, \mathrm{H}-2), 7.40(1 \mathrm{H}, \mathrm{dd}, J=3.6,15.7, \mathrm{H}-3) .{ }^{13} \mathrm{C}-\mathrm{NMR}\left(75 \mathrm{MHz}, \mathrm{CDCl}_{3}, \delta\right.$, ppm) 20.7 (q, C-16), 26.4 (t, C-13), 31.6 (t, C-12), 34.3 (t, C-14), 42.5 (t, C-6), 44.8 (t, C-8), 46.5 (d, C-9), 49.9 (d, C-5), 71.7 (d, C15), 76.6 (d, C-4), 118.5 (d, C-2), 132.3 (d, C-11), 135.1 (d, C-10), 150.3 (d, C-3), 166.1 (s, C-1), 216.2 (s, C-7). Mass spectrum (HR-ESITOF-MS) m/z: 301.1409 (Calcd for $\mathrm{C}_{16} \mathrm{H}_{22} \mathrm{O}_{4} \mathrm{Na}$ : 301.1410).

5,6-Dehydro-2,3-dihydro-4,7-dioxo-BFA (12) Yield $42 \%$. ${ }^{1} \mathrm{H}-\mathrm{NMR}(300 \mathrm{MHz}, \mathrm{CDCl} 3, \delta$, ppm, $J / \mathrm{Hz}) 0.90$ (1H, m, H-13a), 1.26 (3H, d, $J=6.3, \mathrm{H}-16), 1.43-1.81$ (4H, m, H-12a, H-13b, H-14), 2.06 $(1 \mathrm{H}, \mathrm{m}, \mathrm{H}-12 \mathrm{~b}), 2.31$ (1H, dd, $J=1.8,19.2, \mathrm{H}-8 \mathrm{a}), 2.55$ (1H, m, H-2a), $2.70-3.07$ (3H, m, H-2b, H-3a, H-8b), 3.12 (1H, m, H-3b), 3.93 (1H, br. t, $J=7.6, \mathrm{H}-9), 4.85$ (1H, m, H-15), 5.22 (1H, dd, $J=8.4,15.3$, $\mathrm{H}-10), 5.56(1 \mathrm{H}, \mathrm{ddd}, J=6.9,8.1,15.1, \mathrm{H}-11), 6.48(1 \mathrm{H}, \mathrm{d}, J=1.4, \mathrm{H}-6) .{ }^{13} \mathrm{C}-\mathrm{NMR}\left(75 \mathrm{MHz}, \mathrm{CDCl}_{3}, \delta\right.$, ppm) 18.7 (q, C-16), 22.6 (t, C-13), 29.5 (t, C-2), 30.6 (t, C-12), 32.5 (t, C-14), 36.4 (t, C-3), 42.5 (t, C-8), 42.8 (d, C-9), 71.3 (d, C-15), 129.4 (d, C-11), 133.9 (d, C-10), 134.1 (d, C-6), 156.3 (s, C-5), 172.1 (s, C1), 199.1 (s, C-7), 208.4 (s, C-4). Mass spectrum (HR-ESITOF-MS) m/z: 299.1262 (Calcd for $\left.\mathrm{C}_{16} \mathrm{H}_{20} \mathrm{O}_{4} \mathrm{Na}: 299.1254\right)$.

\section{Synthesis of tetrahydroBFA (13)}

BFA (1, $10 \mathrm{mg})$ in methanol $(1 \mathrm{~mL})$ was hydrogenated over $\mathrm{Pd} / \mathrm{C}$ for $4 \mathrm{~h}$. The filtrate was evaporated and the residue was chromatographed by elution with $\mathrm{CH}_{2} \mathrm{Cl}_{2} / \mathrm{MeOH}$ (99:1) to afford tetrahydroBFA $(\mathbf{1 3}, 8.3 \mathrm{mg}, 82 \%)$.

TetrahydroBFA (13) Yield $82 \% .{ }^{1} \mathrm{H}-\mathrm{NMR}\left(300 \mathrm{MHz}, \mathrm{CDCl}_{3}, \delta\right.$, ppm, J/Hz) $0.90(1 \mathrm{H}, \mathrm{m}, \mathrm{H}-13 \mathrm{a})$, $1.25(3 \mathrm{H}, \mathrm{d}, J=6.3, \mathrm{H}-16), 1.29-1.56(8 \mathrm{H}, \mathrm{m}, \mathrm{H}-8 \mathrm{a}, \mathrm{H}-11, \mathrm{H}-12, \mathrm{H}-13 \mathrm{~b}, \mathrm{H}-14), 1.63-2.00$ (8H, m, H-3, H-5, H-6, H-9, H-10), 2.11 (1H, m, H-8b), 2.45 (2H, m, H-2), 3.84 (1H, m, H-4), 4.30 (1H, m, H-7), 4.94 $(1 \mathrm{H}, \mathrm{m}, \mathrm{H}-15) .{ }^{13} \mathrm{C}-\mathrm{NMR}\left(75 \mathrm{MHz}, \mathrm{CDCl}_{3}, \delta\right.$, ppm) 20.0 (q, C-16), 22.5 (t, C-13), 24.6 (t, C-11), $27.3(\mathrm{t}$, C-12), 31.8 (t, C-10), 32.3 (t, C-2), 32.5 (t, C-14), 34.0 (t, C-3), 36.0 (t, C-6), 38.6 (d, C-9), 41.8 (t, C-8), 47.5 (d, C-5), 72.0 (d, C-15), 72.9 (d, C-7), 73.0 (d, C-4), 176.3 (s, C-1). Mass spectrum (HR-ESITOFMS) $m / z: 307.1879$ (Calcd for $\mathrm{C}_{16} \mathrm{H}_{28} \mathrm{O}_{4} \mathrm{Na}: 307.1880$ ).

\section{Cytotoxicity assays}

The cytotoxic assays against KB cells (oral human epidermoid carcinoma) and Vero cells (African green monkey kidney fibroblasts) were evaluated using the resazurin microplate assay [31]. The standard drugs were ellipticine and doxorubicin. 


\section{Results and discussion}

The ester analogues were obtained by treating the BFA with an appropriate acid anhydride or acid chloride in pyridine. Apart from the monoacetate analogue, 7-O-acetylBFA (2), which was obtained by refluxing 1 in acetic acid [25], the esters were prepared by treating 1 with an acetic anhydride, propionic anhydride, butyric anhydride, and benzoic anhydride in pyridine to furnish the esters 3-10. Oxidation of BFA with pyridinium chlorochromate selectively yielded the mono-oxo analogue 11, while prolonged oxidation resulted in the formation of the di-oxo analogue 12 [25]. The carbon-carbon double bonds of BFA were hydrogenated to yield tetrahydro BFA (13).

All synthetic compounds were purified by column chromatography, and their structures were established by spectroscopic analysis. For the known compounds, the spectroscopic data were compared with those reported previously. The structure of the analogue $\mathbf{2}$ could be distinguished from that of $\mathbf{3}$ by the relatively downfield shift of H-7 and H-4 in the former and the latter, respectively. The structure of the oxidation product of $\mathbf{1}$ was assigned as $\mathbf{1 1}$ based on the disappearance of $\mathrm{H}-7$ signal and the presence of the allylic signal, H-4. The di-oxo analogue 12 was obtained from prolonged oxidation of 1 . The disappearance of the olefinic protons $\mathrm{H}-2, \mathrm{H}-3, \mathrm{H}-10$ and $\mathrm{H}-11$ in $\mathbf{1 3}$ indicated that hydrogenation has taken placed at the 2 and 10-positions.

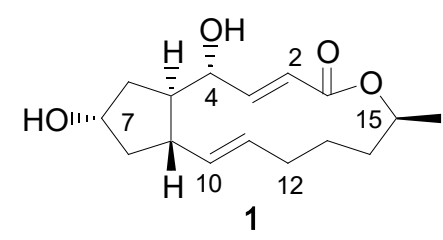

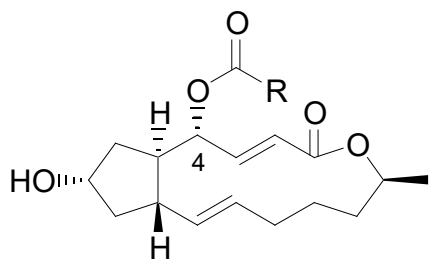

$3 \mathrm{R}=\mathrm{CH}_{3}$

$4 \mathrm{R}=\mathrm{CH}_{2} \mathrm{CH}_{3}$

$5 \mathrm{R}=\left(\mathrm{CH}_{2}\right)_{2} \mathrm{CH}_{3}$

$6 \mathrm{R}=\mathrm{C}_{6} \mathrm{H}_{5}$<smiles>C[C@H](O)/C=C/C(=O)O[C@@H](C)CCC/C=C/[C@H]1CCC(=O)C1</smiles>

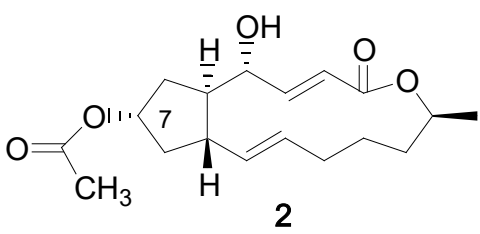

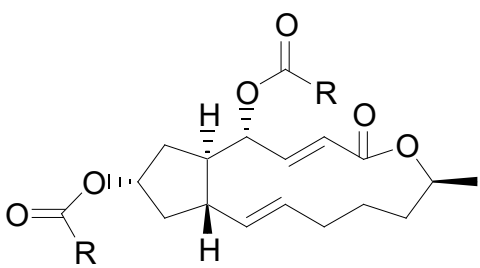

$7 \mathrm{R}=\mathrm{CH}_{3}$

$8 \mathrm{R}=\mathrm{CH}_{2} \mathrm{CH}_{3}$

$9 \mathrm{R}=\left(\mathrm{CH}_{2}\right)_{2} \mathrm{CH}_{3}$

$10 \mathrm{R}=\mathrm{C}_{6} \mathrm{H}_{5}$<smiles>[H]/C=C\[C@H]1CC(=O)C=C1C(=O)CC/C=C\C(=O)OC(C)C</smiles><smiles>C[C@@H]1[CH][C@@H](C(O)CCC(=O)O[C@@H]2C[C@@H](O)C[C@@H]2O)CCCCC1</smiles>

Figure 1 Chemical Structures of Compounds 1-13.

The compounds 1-13 were the first report of the cytotoxic against human epidermoid carcinoma (KB) cells as shown in Table 1. The macrolide $\mathbf{1}$ exhibited very strong anticancer activity against KB cells with the $\mathrm{IC}_{50}$ of $0.0023 \mu \mathrm{M}$. However, compound 1 was very toxic against the Vero cell with an $\mathrm{IC}_{50}$ of $0.082 \mu \mathrm{M}$. For the KB cytotoxicity, the monoacetate analogue 2 was exceptionally highly active, with the $\mathrm{IC}_{50}$ of $0.000034 \mu \mathrm{M}$, or 68 -fold more active than the parent compound 1 . It was $41764-$ and 8235 fold more active than ellipticine and doxorubicin, respectively. It has been reported that acetyl BFAs 
exhibited nearly equivalent activities with BFA with respect to cytotoxicity and induction of apoptosis in the human colon cancer cell HCT116 [24]. In this study, we found that the 7-O-acetyl BFA (2) possessed the very high cytotoxicity against $\mathrm{KB}$ cells with the $\mathrm{IC}_{50}$ of $0.000034 \mu \mathrm{M}$ and very high selectivity index of 9117. The difference in the effect of acetylation might depend on the cells used. The results indicated that the aliphatic chain extended monoderivatives (3-5) and increased cytotoxic against KB cell lines. A similar trend is seen with the diderivative analogues for compounds (7-9). This may be due to the steric bulk in the binding site(s). The analogues 3-5 and 7-9, though less active than their parent compound 1, exhibited cytotoxicity against the KB cells in submicromolar order of magnitude.

The monobenzoyl ester 6 was the second most active analogue with the $\mathrm{IC}_{50}$ of $0.0052 \mu \mathrm{M}$. Despite its approximately 2-fold less active than the parent compound 1, the analogue 6 was 59-fold less toxic against the Vero cells than the parent compound 1. This resulted in an outstanding selectivity index of 925. The remaining compounds, 10 and 11-13, were less active than 1. Oxidation and hydrogenation of BFA resulted in a significant loss of activity. This is consistent with the previously reported study [24]. The results have indicated that the 4- $O$-ester analogues (compounds 3-6) were less active than the parent compound 1. For the alkyl ester analogues, the length of the alkyl groups did not seem to affect the cytotoxic activity of the analogues. Addition of the second alkyl ester group to the 7-position resulted in 2- to 5-fold increase in activity. These results suggest that 4-hydroxyl group is more important than 7hydroxyl group for cytotoxicity against $\mathrm{KB}$ cells. It could be concluded that the essential structural features for the macrolide of BFA-type to exhibit high cytotoxicity against KB cells are the presence of a free hydroxyl group at the 4-position, a free hydroxyl group or the corresponding ester at the 7-position, and unsaturated functions at the 2- and 10-positions.

Table 1 Cytotoxic Activities of Compounds 1-13 $\left(\mathrm{IC}_{50}, \mu \mathrm{M}\right)$.

\begin{tabular}{cccc}
\hline \multirow{2}{*}{ Compounds } & \multicolumn{3}{c}{ Cytotoxicity, $\mathbf{I C}_{\mathbf{5 0}}[\boldsymbol{\mu M}]$} \\
\cline { 2 - 4 } $\mathbf{1}$ & $\mathbf{K B}^{\mathbf{a}}$ & $\mathbf{S I}^{\mathbf{b}}$ & Vero cells \\
$\mathbf{2}$ & 0.0023 & 35.65 & 0.082 \\
$\mathbf{3}$ & 0.000034 & 9117 & 0.31 \\
$\mathbf{4}$ & 0.081 & 41.36 & 3.35 \\
$\mathbf{5}$ & 0.054 & 270.74 & 14.62 \\
$\mathbf{6}$ & 0.023 & 166.09 & 3.82 \\
$\mathbf{7}$ & 0.0052 & 925 & 4.81 \\
$\mathbf{8}$ & 0.030 & 738 & 22.14 \\
$\mathbf{9}$ & 0.010 & 779 & 7.79 \\
$\mathbf{1 0}$ & 0.0095 & 645.26 & 6.13 \\
$\mathbf{1 1}$ & 2.19 & 35.99 & 78.82 \\
$\mathbf{1 2}$ & 71.75 & 0.61 & 43.54 \\
$\mathbf{1 3}$ & inactive & - & - \\
Ellipticine & 1.64 & 5.83 & 9.56 \\
Doxorubicin & 1.42 & 3.37 & 4.78 \\
\hline & 0.28 & - & - \\
\hline
\end{tabular}

\footnotetext{
${ }^{\text {a }}$ Human epidermoid carcinoma of the mouth

${ }^{\mathrm{b}}$ Selectivity index.
} 


\section{Conclusions}

In conclusion, the mono and diester analogues of BFA were synthesized and evaluated for cytotoxicity against KB cells. Most of the analogues had potent cytotoxicity against the cancer cell lines, whereas significant decrease in cytotoxicity against the Vero cells was observed. The parent compound $\mathbf{1}$ and most of the analogues were more potent than ellipticine and doxorubicin against cancer cell line tested. 7-O-AcetylBFA (2) possessed the highest activity against $\mathrm{KB}$ cells. A slight increase in cytotoxicity was observed with the increase in the alkyl chain length of the monoester analogues. An increased in activity was noted in going from the monoalkyl ester analogues to dialkyl ester analogues. The presence of aromatic ester moiety resulted in the decrease of the activity. Many of the analogues showed very high selectivity index. These results suggested that the analogues might be utilized for the development of new candidates for the treatment of cancer. Therefore, further development of such compounds may be of interest.

\section{Acknowledgements}

This work was supported by the Center of Excellence for Innovation in Chemistry (PERCH-CIC), Ministry of Higher Education, Science, Research and Innovation, and Srinakharinwirot University. Partial support from The Thailand Research Fund (DBG6180030) is gratefully acknowledged. We are indebted to the Bioassay Research Facility of the National Center for Genetic Engineering and Biotechnology (BIOTEC) for bioactivity tests and Chulabhorn Research Institute, Bangkok, for recording the high resolution mass spectra.

\section{References}

[1] VL Singleton, N Bohonos and AJ Ullstrup. Decumbin, a new compound from a species of Penicillium. Nature 1958; 181, 1072-3.

[2] E Haerri, W Loeffler, HP Sigg, H Stähelin and C Tamm. Über die isolierung neuer Stoffwechselprodukte aus Penicillium brefeldianum DODGE. Helv. Chim. Acta 1963; 46, 1235-43.

[3] GP Miao, J Han, KG Zhang, SC Wang and CR Wang. Protection of melon against Fusarium wiltroot knot nematode complex by endophytic fungi Penicillium brefeldianum HS-1. Symbiosis 2019; 77, 83-9.

[4] V Betina, P Nemec, J Dobias and Z Baráth. Cyanein, a new antibiotic from Penicillium cyaneum. Folia. Microbiol. 1962; 7, 353-7.

[5] Y Suzuki, H Tanaka, H Aoki and T Tamura. Ascotoxin (decumbin), a metabolite of Ascochyta imperfecta PECK. Agr. Biol. Chem. 1970; 34, 395-413.

[6] M Chinworrungsee, S Wiyakrutta, N Sriubolmas, P Chuailua and A Suksamrarn. Cytotoxic activities of trichothecenes isolated from an endophytic fungus belonging to order hypocreales. Arch. Pharm. Res. 2008; 31, 611.

[7] K Trisuwan, V Rukachaisirikul, Y Sukpondma, S Phongpaichit, S Preedanon and J Sakayaroj. Lactone derivatives from the marine-derived fungus Penicillium sp. PSU-F44. Chem. Pharm. Bull. 2009; 57, 1100-2.

[8] YJ Wang, YF Wu, F Xue, ZX Wu, YP Xue, YG Zheng and YC Shen. Isolation of brefeldin A from Eupenicillium brefeldianum broth using macroporous resin adsorption chromatography. $J$. Chromatogr. B 2012; 895-896, 146-53.

[9] Z Hu, L Qin, W Ding, Y Liu and Z Ma. New analogues of brefeldin A from sediment-derived fungus Penicillium sp. DT-F29. Nat. Prod. Res. 2016; 30, 2311-5.

[10] J Xie, YY Wu, TY Zhang, MY Zhang, WW Zhu, EA Gullen, ZJ Wang, YC Cheng and YX Zhang. New and bioactive natural products from an endophyte of Panax notoginseng. RSC Adv. 2017; 7 , 38100-9.

[11] V Betina. Biological effects of the antibiotic brefeldin A (Decumbin, cyanein, ascotoxin, synergisidin): A retrospective. Folia Microbiol. 1992; 37, 3-11.

[12] L Zhou, W Gao, K Wang, Z Huang, L Zhang, Z Zhang, J Zhou, EC Nice and C Huang. Brefeldin A inhibits colorectal cancer growth by triggering Bip/Akt-regulated autophagy. FASEB J. 2019; 33, 5520-34.

[13] H Huang, T Liu, J Guo, L Yu, X Wu, Y He, D Li, J Liu, K Zhang, X Zheng and S Goodin. Brefeldin A enhances docetaxel-induced growth inhibition and apoptosis in prostate cancer cells in monolayer and 3D cultures. Bioorg. Med. Chem. Lett. 2017; 27, 2286-91. 
[14] SA Lee, YJ Kim and CS Lee. Brefeldin A induces apoptosis by activating the mitochondrial and death receptor pathways and inhibits focal adhesion kinase-mediated cell invasion. Basic Clin. Pharmacol. Toxicol. 2013; 113, 329-38.

[15] AB Argade, R Devraj, JA Vroman, RD Haugwitz, M Hollingshead and M Cushman. Design and synthesis of Brefeldin A sulfide derivatives as prodrug candidates with enhanced aqueous solubilities. J. Med. Chem. 1998; 41, 3337-46.

[16] AB Argade, RD Haugwitz, R Devraj, J Kozlowski, PE Fanwick and M Cushman. Highly efficient diastereoselective michael addition of various thiols to (+)-Brefeldin A. J. Org. Chem. 1998; 63, 273-8.

[17] BM Fox, JA Vroman, PE Fanwick and M Cushman. Preparation and evaluation of sulfide derivatives of the antibiotic Brefeldin A as potential prodrug candidates with enhanced aqueous solubilities. J. Med. Chem. 2001; 44, 3915-24.

[18] L Renault, B Guibert and J Cherfils. Structural snapshots of the mechanism and inhibition of a guanine nucleotide exchange factor. Nature 2003; 426, 525-30.

[19] Y Pommier and J Cherfils. Interfacial inhibition of macromolecular interactions: Nature's paradigm for drug discovery. Trends Pharmacol. Sci. 2005; 26,138-45.

[20] H Sung, J Ferlay, RL Siegel, M Laversanne, I Soerjomataram, A Jemal and F Bray. Global cancer statistics 2020: GLOBOCAN estimates of incidence and mortality worldwide for 36 cancers in 185 countries. CA Cancer J. Clin. 2021; 71, 209-49.

[21] World Health Organization. The global cancer observatory (GCO), Available at: https:/gco.iarc.fr/today/data/factsheets/populations/764-thailand-fact-sheets.pdf, accessed May 2021.

[22] A Miranda-Filho and F Bray. Global patterns and trends in cancers of the lip, tongue and mouth. Oral Oncol. 2020; 102, 104551.

[23] JW Zhu, H Hori, H Nojiri, T Tsukuda and Z Taira. Synthesis and activity of Brefeldin A analogs as inducers of cancer cell differentiation and apoptosis. Bioorg. Med. Chem. Lett. 1997; 7, 139-44.

[24] JW Zhu, H Nagasawa, F Nagura, SB Mohamad, Y Uto, K Ohkura and H Hori. Elucidation of strict structural requirements of Brefeldin A as an inducer of differentiation and apoptosis. Bioorg. Med. Chem. 2000; 8, 455-63.

[25] B Proksa, D Uhrin, J Adamcova and J Fuska. Oxidation of Brefeldin A. Pharmazie 1992; 47, 582-4.

[26] M Shibazaki, H Yamaguchi, T Sugawara, K Suzuki and T Yamamoyo. Microbial glycosylation and acetylation of Brefeldin A. J. Biosci. Bioeng. 2003; 96, 344-8.

[27] NO Anadu, VJ Davisson and M Cushman. Synthesis and anticancer activity of Brefeldin A ester derivatives. J. Med. Chem. 2006; 49, 3897-905.

[28] K Tian, F Xu, X Gao, T Han, J Li, H Pan, L Zang, D Li, Z Li and T Uchita. Nitric oxide-releasing derivatives of Brefeldin A as potent and highly selective anticancer agents. Eur. J. Med. Chem. 2017; 136, 131-43.

[29] B He, Y Wang, Y Zheng, W Chen and Q Zhu. Synthesis and cytotoxic evaluation of acylated Brefeldin A derivatives as potential anticancer agents. Chem. Biol. Drug. Des. 2013; 82, 307-16.

[30] K Seehafer, F Rominger, G Helmchen, M Langhans, DG Robinson, B Oezata, B Bruegger, JR Strating, FJ van Kuppeveld and CD Klein. Synthesis and biological properties of novel Brefeldin A analogues. J. Med. Chem. 2013; 56, 5872-84.

[31] J O'Brien, I Wilson, T Orton and F Pognan. Investigation of the alamar blue (resazurin) fluorescent dye for the assessment of mammalian cell cytotoxicity. Eur. J. Biochem. 2000; 267, 5421-6. 